\title{
The Simulation Study of the Locking Device in Platform Screen Door System
}

\author{
Haiying Zhang ${ }^{1 \mathrm{a}}$, Weiyan $\mathrm{Xu}^{1 \mathrm{~b}^{*}}$ and Xiangyan $\mathrm{Yu}^{2, \mathrm{c}}$ \\ ${ }^{1}$ Qingdao Binhai University, Qingdao, China, 266555 \\ ${ }^{2}$ Qingdao Qian wan Container Terminal Co., Ltd., Qingdao, China, 266550 \\ a64029205@qq.com, bzhanghy_ok@sina.com, '8311556@qq.com
}

Keywords: Platform screen door system; Solid modeling; Simulation

\begin{abstract}
Describes. the automatic door locking door system structure and function, and in Pro/E environment, the locking device of the solid modeling, virtual assembly and interference checking, assembly of components of the locking-device for three-dimensional design fork, further exploration of virtual prototyping and dynamic simulation technology in the mechanical design applications, virtual assembly simulation help with early detection and intervention to avoid the process of product design and assembly defects in order to ensure the locking device in the actual production assembly feasibility, simplify the mechanical design of the product development process, significantly reducing the product development cycle.
\end{abstract}

\section{Introduction}

Door locking device is an important part of the automatic door system, with the advances in automation technology, automatic door lock door system is widely used in banks, airports waiting for planes hall, subway level of sliding doors and other special places, some of its zero different occasions by the installation of parts to change. Door locking device function and structure determines the reliability of the automatic door system security, and its shape, size and dimensions directly affect the normal operation of door effect. At present, the door locking device is designed to develop general design is the first, during the intensity calibration, and structural design. As the design process to consider the actual situation is more complex, often to the final assembly stage of the product only to find design problems, such as the design size is too large and cannot cause interference in the assembly, the resulting product is difficult to guarantee the quality of assembly and other issues [1]. In the Pro /E environment for the door lock device body solid modeling, virtual assembly and interference checking, its structural design has an important significance.

\section{The Door Locking Device Structure and Working Principle}

Horizontal sliding doors with automatic locks, automatically unlock and "in place and lock the door" test and manual unlocking functions. Door locking device installed above the door in the middle of automatic doors, work, and motor driven door in the locked position by the locking action to complete the locking hook, completed by the solenoid to unlock. Automatic door lock system, door installations where some of its parts because of different changes. The device consists of frame body, the lock hook, fork, shaft, automatic unlock institutions, signal detection agencies, reset mechanism, the reverse body and manual unlocking institutions (see Fig, 1, Fig. 2). 


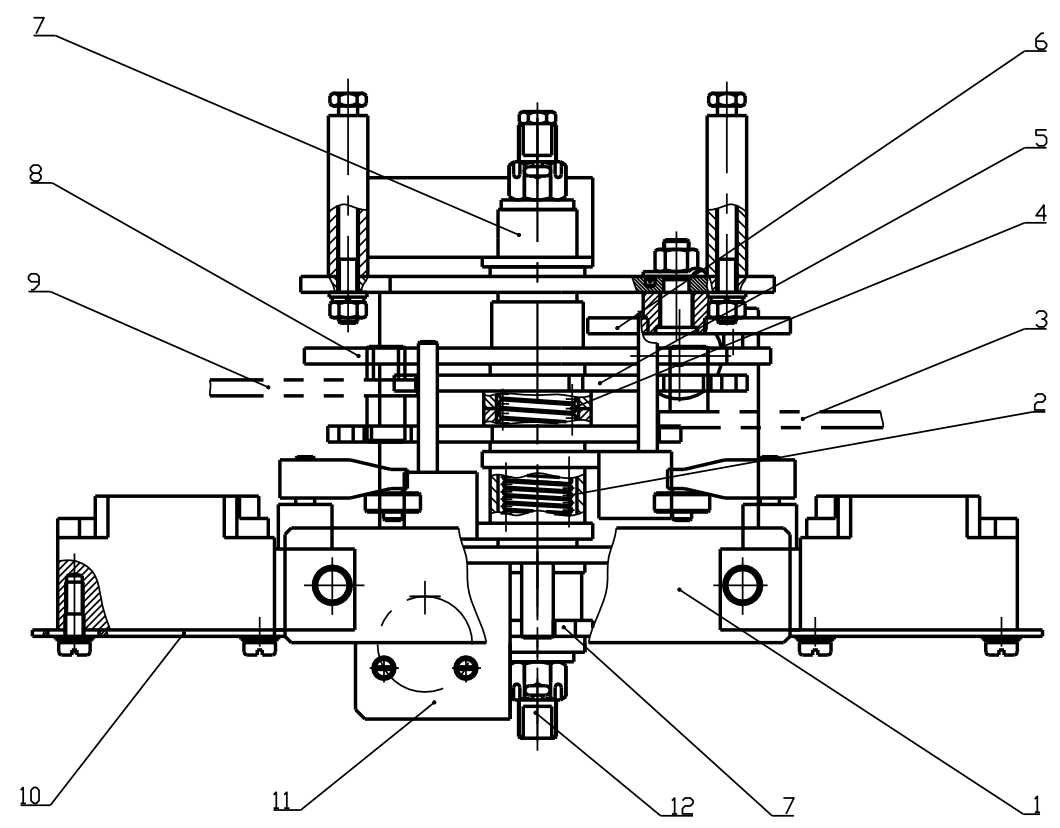

Figure 1. Structural diagram of door lock device

1. Bracket 2. reset thick torsion spring 3. The right door plate 4. reset thin torsion spring 5 . The fork 6.everse body 7. the manual unlocking institutions 8 . The lock hook 9. The left door plate 10.signal detection agencies 11.automatically unlock institutions 12 . Shaft

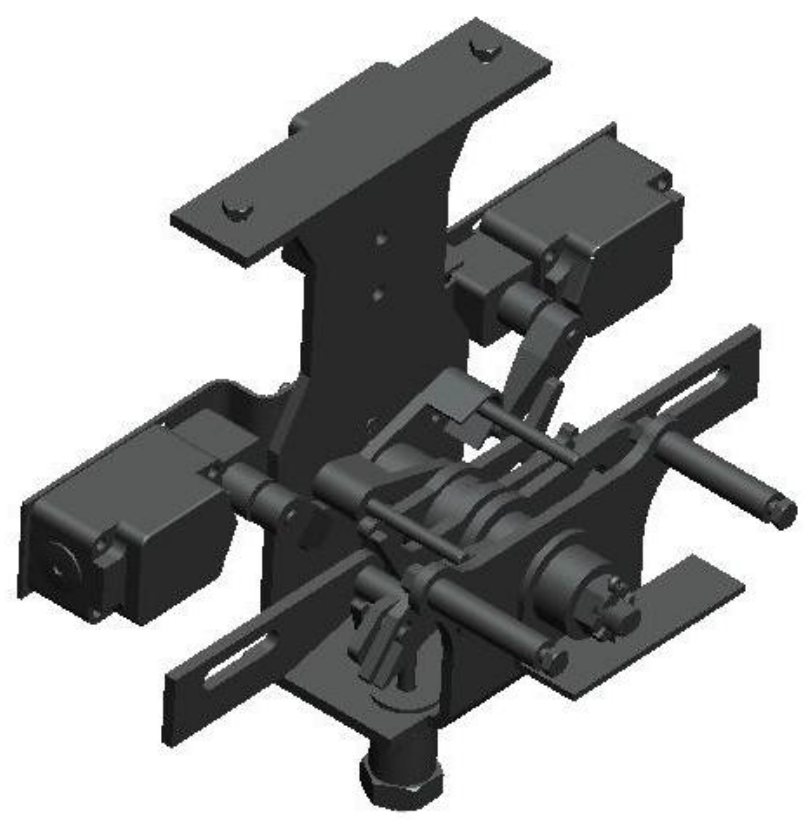

Figure 2. Three-dimensional shape of the body locking device map

\section{Application of Pro / E Solid Modeling, Virtual Assembly and Interference Checking}

Door locking devices such as mechanical design, in the past have used two-dimensional graphics, it is difficult to achieve parameters, serial, difficult to analyze and reflect the various components and the relationship between the assembly machine. The three-dimensional computer simulation design technology in recent years with the rise of the rapid development of new technologies. Three-dimensional simulation design technology, computer-based power, the CAD/CAE/CAM technology to product design, so that designers in the product design stage can be completed on the computer and virtual manufacturing of products of the product be modified to achieve optimal product design. 
Pro/E is currently the most popular 3D CAD software is one of the feature-based, take Parametric Technology, all data related to a single integrated database to support concurrent engineering parameters of the physical operation of the latest design software. The adopted parametric feature modeling technology, is suitable for automatic door system door lock device simulation application requirements. The use of Pro/E software for the automatic door locking system, door solid modeling process (see Fig. 3).

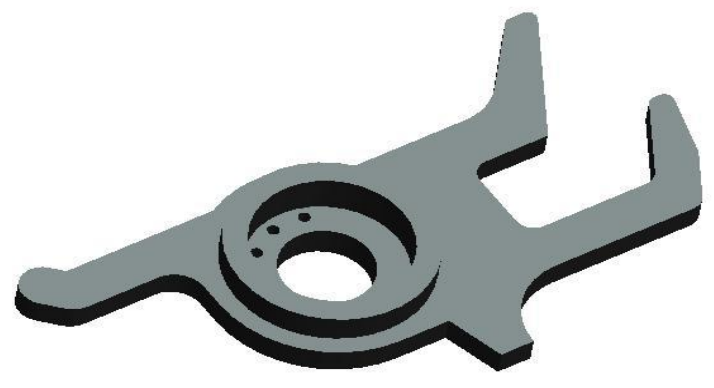

Figure 3

\section{Creating the Characteristics of Individual Parts}

Pro/E is a geometric model of the main features of the system, for data access is to feature as the smallest units, all of the parameters are based on completion of a feature created for the purpose, so each part by a series of characteristics of the composition, and each feature will change the part geometry, part geometry and can add some design information

Model for the design of parts, the first part of the characteristics of planning, down to its multiple features, through a series of physical characteristics or surface features to create, step by step complete part model. General form of the main features of selected parts or features of a larger size as the basic features of the rest as an auxiliary feature, by stretching, cutting and other operations to modify the main features of the last complete some part of the overall shape of the impact of small, but more likely to cause the failure of parameter-driven decorative features such as chamfers, fillets, etc

To fork, for example, the first by stretching a special polyhedron formed the basic shape, and through stretch, array functions complete removal of the hollow cylindrical shape and internal holes, etc.. Finishing chamfers and fillets, to complete parts of the 3D model (see Fig. 4).

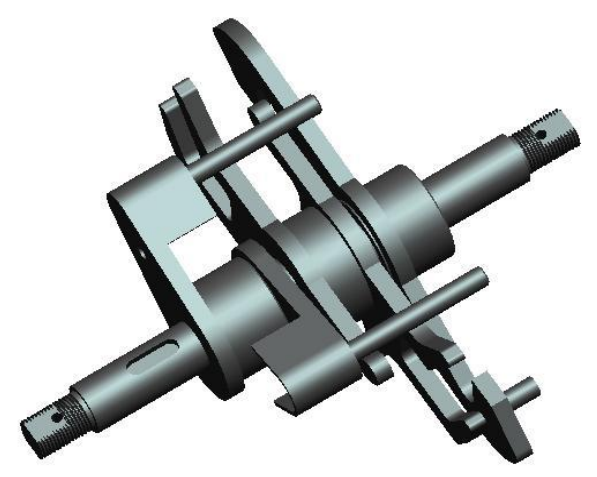

Figure 4. Three-dimensional modeling of flow body locking device 


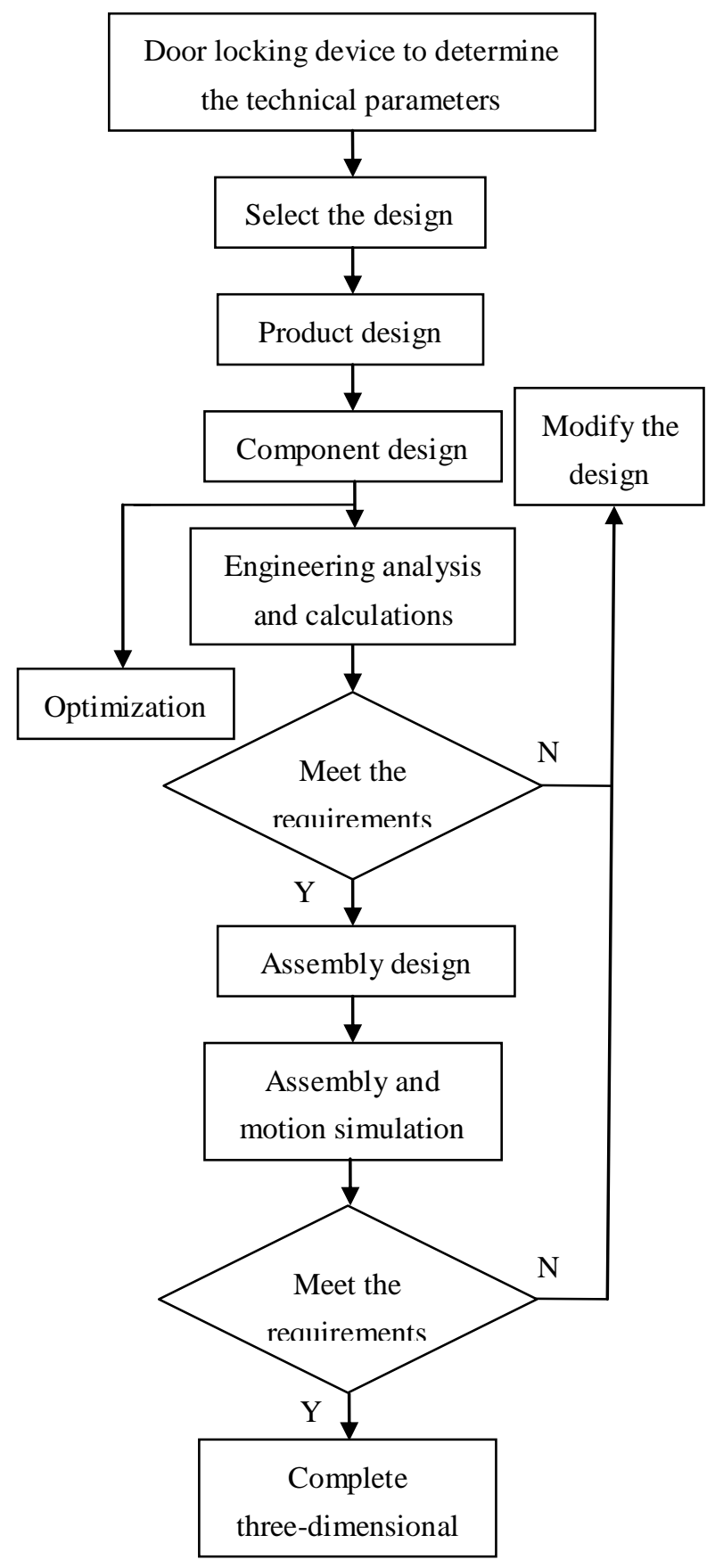

Figure 5. lock hook - shaft sub-assembly modeling diagram

\section{Virtual Assembly}

Virtual assembly based on physical models of digital products, computer products on the assembly of the analysis and verification performance, to improve the product can be assembled to provide a strong support. Virtual assembly technology, can facilitate the realization of the assembly process simulation, verification and analysis for assembly, assembly process planning, etc., so more and more enterprises will be used in virtual assembly technology mechanical product development.

Pro/E components of the module provides a virtual assembly capabilities. In the Pro/E in an assembly called by a series of parts or components (Component) and the component or assembly, said (Assembling) in accordance with a certain combination of location and binding relationship together.

Automatic door system of the machine door locking device and sub-assembly design process is: first mediator matrix components, the so-called base model of the entire assembly parts is the most 
critical part is the assembly of the base, and its existence will affect parts subsequently transferred. Secondly transferred to the relevant part model. Finally, using a variety of constraints on the location of each part to the direction of the constraints to complete the assembly design.

To lock hook - shaft sub-assembly, for example (see Figure 5), the base part of the shaft, the other parts including the lock hook, the left fork and the left arm and so on. In the formation of the sub-assembly, the first import shaft, the assembly will be the basis of their orientation to the three planes, and then use it as a base part, the use of matching, alignment, and other constraints into the relationship between the assembly transferred to other parts. The final completion of the assembly model.

\section{Motion Simulation and Interference Checking}

Interference checking is designed to test the parts in the assembly of the final position of the relative positions of the existence of interference, to ensure the movement of components in the product does not work with the surrounding parts collision interference. In the assembly process to be conducted for each assembly component interference checking, if there is interference in the case, the design must be modified to satisfy the needs with the requirements of the assembly.

Use of Pro/E door locking device from the body parts to the overall design, complete door locking devices and assembled three-dimensional solid model simulation, we can be rotated, a different perspective from the various agencies to see if in motion interference. Just change the body parts in the assembly constraint relationship, you can drag the mouse so that the components without constraints on the freedom of movement or rotation. This realization of the assembly of dynamic simulation.

Also, you can use Pro/MECHANICA simulation analysis module, the Pro/E in the model and its link, the full realization of geometric modeling and finite element analysis integration.

\section{Conclusions}

In this paper, Pro/E environment to achieve the automatic door system door locking device for three-dimensional solid modeling, virtual assembly, interference testing. Virtual assembly and collision detection will help in early detection and to avoid product design and assembly defects in the process, through the automatic door system, door locking device of the functional design, 3D modeling and simulation on the computer to establish a virtual prototype model simplify the mechanical design of the product development process, significantly reducing the product development cycle. A significant reduction in product development costs and costs for computer-aided design technology in the modern mechanical design to explore the development and application of new methods and ideas.

\section{References}

[1] Tu Zhi ping, Li Zhi jie, virtual assembly and body motion simulation applications on the gear unit in the vehicle [J]. Railway communication signal, (2010),46 (1) :4-7

[2] National Cheng Kung University first. Mechanical Design Handbook, Beijing: Chemical Industry Press, (2014)

[3] Lin Qingan. Pro / ENGINEER Wildfire 2.0. Tsinghua University Press, (2012)

[4] Tangzi Chun. PLC Principle and Application of Technology [M] Beijing: Higher Education Press, (2013):15-25.

[5] Sun Zhen qiang. Programmable controllers' theory and application tutorial [M].2, Beijing: Qinghua University Press. (2013):41-44.

[6] Liao chang chu. Programmable controller application technology [M].2 version. Chongqing: Chongqing University Press. (2014):52-70. 
[7] Zhang Wanzhong. PLC application and maintenance techniques [M]. Beijing: Chemical Industry Press. (2012):7-22.

[8] Wang Daohui editor. Logic and programmable control systems [M]. Beijing Machinery Industry Press, (2010)

[9] Shao Yubin .Matlab / Simulink modeling and simulation of communication systems case study [M]. Beijing: National Defense Industry Press, (2012). 